
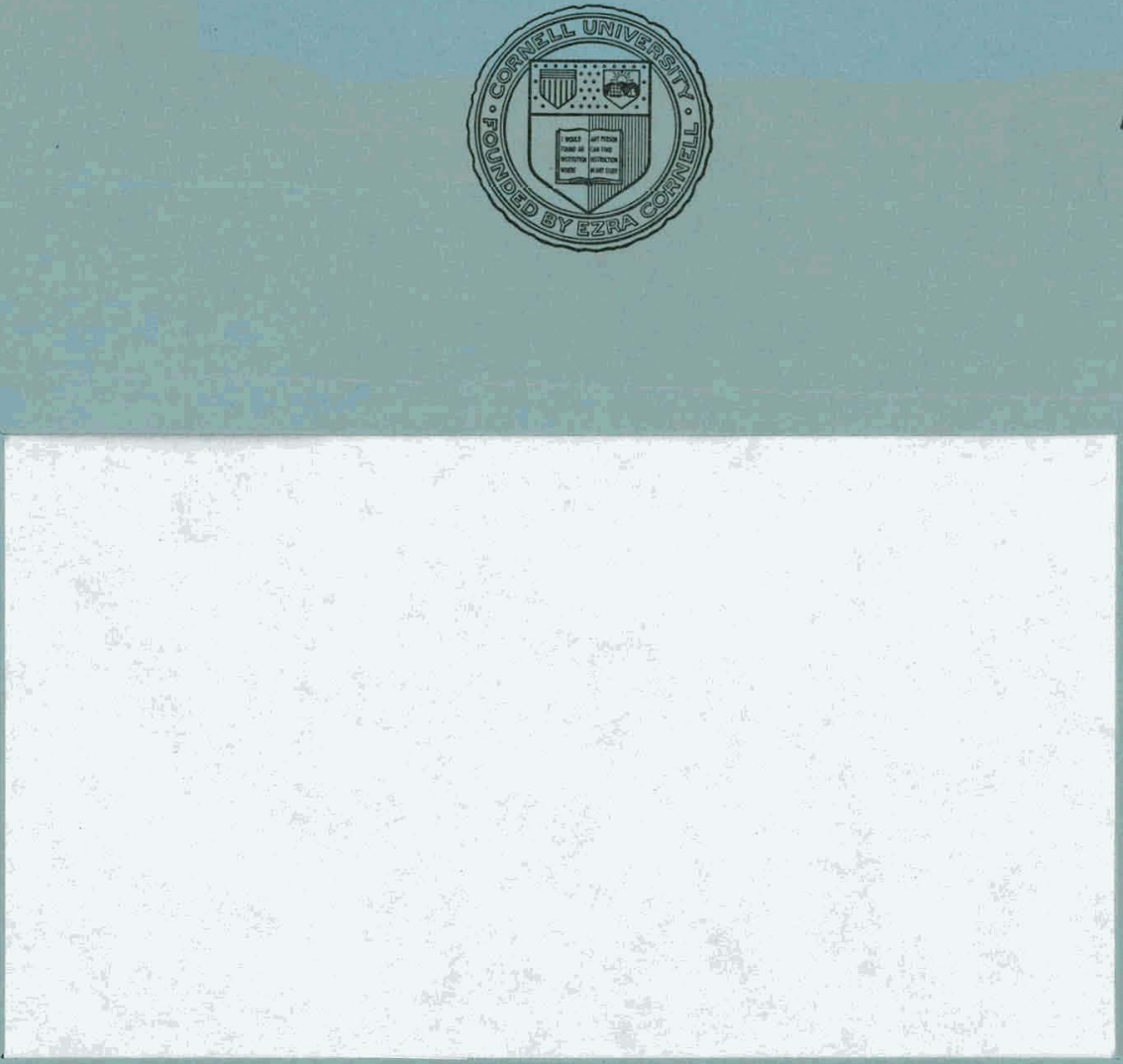

Department of

Theoretical and Applied Mechanics

CORNELL. UNIVERSITY

ITHACA, NEW YORK 


\section{DISCLAIMER}

This report was prepared as an account of work sponsored by an agency of the United States Government. Neither the United States Government nor any agency Thereof, nor any of their employees, makes any warranty, express or implied, or assumes any legal liability or responsibility for the accuracy, completeness, or usefulness of any information, apparatus, product, or process disclosed, or represents that its use would not infringe privately owned rights. Reference herein to any specific commercial product, process, or service by trade name, trademark, manufacturer, or otherwise does not necessarily constitute or imply its endorsement, recommendation, or favoring by the United States Government or any agency thereof. The views and opinions of authors expressed herein do not necessarily state or reflect those of the United States Government or any agency thereof. 


\section{DISCLAIMER}

Portions of this document may be illegible in electronic image products. Images are produced from the best available original document. 


\title{
BOUNDARY ELEMENT ANALYSIS \\ OF TTME-DEPENDENT INELASTIC DEFORMATION \\ OF CRACKED PLATES \\ LOADED IN ANTI-PLANE SHEAR
}

\author{
SUBRATA MUKHERJEE \\ MAHESH MORJARIA
}

\section{Department of Theoretical and Applied Mechanics \\ Thurston Ha11, Cornel1 University \\ Ithaca, NY 14853, U.S.A.}

DOE Report No. DOE/ER/2733-28

\author{
Prepared by Corne1.1 University \\ Under Department of Energy \\ Contract No. EG-77-S-02-2733
}

\section{July 1980}

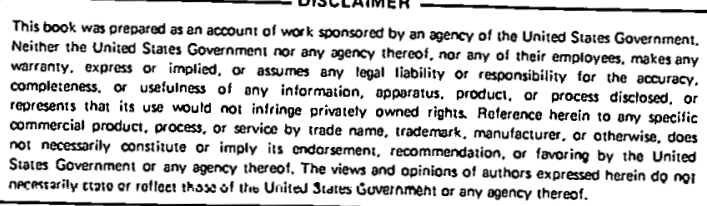

Siates Government of any agency thereat. The views ond opinions of authors expressed herein do not 
A boundary element analysis, for determining stresses in planar cracked bodies undergoing Mode I or Mode II inelastic deformation, has been recentiy published by the present authors $[14,15]$. One primary advantage of this formulation is that the effect of the crack is incorporated in the kernels of the integral equations. Thus, traction free conditions on the crack boundary are satisfied without discretization of this boundary in a numerical calculation procedure.

The inelastic deformation of cracked plates loaded in anti-plane shear (Mode III) is studied in this paper. Modified kernels are used so that the only unknowns in the integral equations are source strengths on the outer boundary of the plate. It is proved that this formulation guarantees traction free cracks as well as single valued displacements on the crack boundary. Numerical results for stresses are presented for various loading histories.with the plate material described by an elastic-nonlinear power law creep constitutive model and a stationary crack modelled as a very thin ellipse. 


\section{INTRODUCTION}

The boundary element method (BEM--also called the boundary-integral equation method) has been gathering momentum in recent years as a powerful general purpose method for the solution of problems in engineering science (see, for example, refs. [1-4] which mainly contain mechanics related applications): The present authors, together with others, have been interested for some time in the application of the BEM to problems of time-dependent inelastic deformation [5-10]. Planar and plate bending problems are considered in these papers. The governing differential equations are written in rate form and these are transformed to integral equations, using, as kernels, singular solutions of the equations in an infinite domain.

This approach works well in the problems considered in refs. [5-10] but can present problems if applied to bodies with sharp cutouts or cracks. This is because adequate discrete modelling of crack boundaries usually requires a large number of boundary elements in order to obtain the stresses accurately near crack tips. This number can become prohibitive in some problems and may lead to numerical difficulties. An elegant alternative formulation for planar elastic problems for bodies with cutouts appears in refs. [11-13]. In this approach, the singular kernels for an infinite domain are augmented so that the new kernels are singular solutions for infinite domains with cutouts, with the proper boundary conditions (eg. traction free) satisfied on the cutout surface. Thus, the effect of the cutout on the stress and displacement fields is incorporated in the kernels and discrete modelling of the cutout boundary is no longer necessary.

The principle of linear superposttion is used to advantage in the papers [11-13] in order to dertve the augmented kernels. This principle, of course, is no longer valid for nonlinear inelastic problems. However, the present authors, in two recent publications [14-15], have used this idea to calculate stresses near crack-tips in planar bodies undergoing time-dependent inelastic deformation. 
The governing nonhomogeneous biharmonic equation for the rate of the stress function is transformed into an integral equation using augmented kernels which guarantee traction-free cutouts. Stress histories for cracked plates undergoing Mode I and Mode II deformation have been obtained numerically for various cases and these are presented in ref. [15].

The inelastic deformation of cracked plates loaded in anti-plane shear (Mode III) is the subject of this paper. In this problem the rate of the stress function satisfies Poisson's equation. The paper begins with the well known single layer potential solution for Laplace's equation in a simply connected finite domain. A region with a cutout is considered next and the kernels are augmented so that the new kernels guarantee that the cutout surface is traction free. Explicit kernels for elliptical cutouts are obtained by using a method analogous to the circle theorem of Milne-Thomson [16]. Integral equations for the stress rates are derived and it is proved that this formulation gives single valued displacements on the cutout. These integral equations are solved numerically and numerical results are presented for the time-dependent redistribution of stresses near the crack tips. The plate material is modelled by the elastic-power-law creep constitutive equations and the stationary cracks are modelled as very thin ellipses. These numerical solutions complement analytical solutions for stresses near crack tips in creeping solids obtained by asymptotic methods [17]. However, in this analysis, elastic and nonelastic strains are allowed to coexist near the crack tip. Thus, the complete equations are solved everywhere and there is no need to match near and far field solutions. Also, a single valued displacement field 1s assured on the crack surface. 


\section{GOVERNING DIFFERENTIAL EQUATIONS}

A planar body, with the $x_{1}$ and $x_{2}$ axes in the plane of the body, and the $x_{3}$ axis normal to it, is loaded in anti-plane shear. The nonzero stress components are $\sigma_{31}\left(=\sigma_{13}\right)$ and $\sigma_{32}\left(=\sigma_{23}\right)$ and a stress function $\Phi$ is defined in the usual way such that

$$
\sigma_{31}=\frac{\partial \Phi}{\partial x_{2}}, \sigma_{32}=-\frac{\partial \Phi}{\partial \dot{x}_{1}}
$$

The nonzero strain rates, $\dot{\varepsilon}_{31}$ and $\dot{\varepsilon}_{32}$, are linearly decomposed into the elastic and nonelastic rates, so that the compatibility equation, in rate form, is

$$
\frac{\partial \dot{\varepsilon}_{31}^{e}}{\partial x_{2}}-\frac{\partial \dot{\varepsilon}_{32}^{e}}{\partial x_{1}}=-\left(\frac{\partial \dot{\varepsilon}_{31}^{n}}{\partial x_{2}}-\frac{\partial \dot{\varepsilon}_{32}^{n}}{\partial x_{1}}\right)
$$

Using Hooke's law to write the elastic strain rates in equation (2) in terms of the stress rates, and writing the stress rates in terms of $\Phi$ from equation (1), results in Poisson's equation for the rate of the stress function

$$
\nabla^{2} \dot{\Phi}=-2 G\left[\frac{\partial \dot{\varepsilon}_{31} \cdot}{\partial x_{2}}-\frac{\partial \dot{\varepsilon}_{32}}{\partial x_{1}}\right]
$$

where $\nabla^{2}$ is the Lapiacion opcrator in two-diuenslons and $G$ is the shear modu1us of the material.

Only traction boundary conditions are allowed in this analysis. The traction $\tau_{3} \equiv \tau$ at any point on the boundary of the body equals the tangential derivative of the stress function

$$
\tau=\frac{\mathrm{d} \Phi}{\mathrm{dc}}
$$

where $c$ is the distance measured along a boundary in an anticlockwise sense. 
BOUNDARY ELEMENT FORMULATION

Simply connected body.

The Poisson's equation (3) can be transformed into an integral equation by using a single layer potential

$$
2 \pi \dot{\phi}(p)=\oint_{\partial B}(\operatorname{lns})_{p Q} C(Q) d c_{Q}+\int_{B}(\operatorname{lns}){ }_{p q} c^{(n)}(q) d A_{q}
$$

where $C$ is the boundary density function (or the source strength function in the language of fluid dynamics) to be determined from the boundary conditions, $s$ is the distance between the source point $p$ (or $P$ ) and the field point $q$ (or Q) (where lower case letters denote points inside the body B and capital letters denote points: on its boundary $\partial B$ (Fig. 1)) and

$$
c^{(n)} \equiv-2 G\left(\frac{\partial \dot{\varepsilon}_{31}^{n}}{\partial x_{2}}-\frac{\partial \dot{\varepsilon}_{32}^{n}}{\partial x_{1}}\right)
$$

This equation can alternatively be written in terms of the kernel

$$
K=\operatorname{Re}\left[\hat{\phi}\left(z, z_{0}\right)\right]
$$

as

$$
2 \pi \dot{\Phi}(p)=\oint_{\partial B} K(p, Q) c(Q) d c_{Q}+\int_{B} K(p, q) C^{(n)}(q) d A_{q}
$$

where

$$
\dot{\phi}\left(z, z_{0}\right)=\ln \left(z-z_{0}\right)
$$

Re denotes the real part of the complex argument and $z$ and $z_{0}$ are the source and field points respectively (see Fig. 1).

Body with cutout

Augmented kernels. The singular kernel $\mathrm{K}$ is augmented with regular kernels such that the sum of these guarantee a traction free cutout. Let the cutout boundary 
be $\partial \mathrm{B}_{1}$ (Fig. 2). If the stress function. $\Phi$ is constant on $\partial \mathrm{B}_{1}$, the traction on it is zero by virtue of equation (4). This constant can be taken to be zero without loss of generality.

Suppose first that the cutout is an unit circle. Let a function $\phi^{*}$ be defined as

$$
\phi \cdot\left(\bar{z}, z_{0}\right)=-\ln \left(\frac{1}{z}-z_{0}\right)
$$

This function has the following properties
a) $\phi^{*}$ satisfies Laplace's equation.
b) $\phi^{*}$ is regular outside the unit circle $|z|=1$.
c) $\phi\left(z, \bar{z}, z_{0}\right)=\hat{\phi}\left(z, z_{0}\right)+\phi^{*}\left(\bar{z}, z_{0}\right)=\ln \left(z-z_{0}\right)-\ln \left(\frac{1}{z}-z_{0}\right)$

vanishes on the unit circle $|z|=1$.

Thus, if a kernel defined as the real part of $\phi$ is used in a formulation analogous to equation (6) for a body with a circular cutout, the cutout will be traction free. The derivation of $\phi^{*}$ is analogous to the circle theorem of MilneThomson [15] for two-dimensional irrotational incompressible Inviscid flow of a fluid past a circular cylinder.

The function $\phi^{*}$ for an elliptic cutout is derived by making use of conformal mapping teclutyues. The mapping function

$$
z=w(\xi)=\frac{1}{\xi}+m \xi
$$

transforms the region on and outside an ellipse in the $z$ plane to the region on and inside an unit circle in the $\xi$ plane (see Fig.1 in ref. [15]). The parameter $m=(a-b) /(a+b)$ (with $(a+b) / 2=1$ ) in terms of the semimajor and minor axes, $a$ and $b$ respectively, of the ellipse. Thus, $m=1$ represents a line crack. Now 


$$
\hat{\phi}=\ln \left(z-z_{0}\right)=\ln \left(\frac{1}{\xi}+m \xi-z_{0}\right)=\ln m+\ln \left(\xi-r_{0}\right)+\ln \left(1-r_{i} / \xi\right)
$$

where $r_{0, i}=\frac{z_{0} \pm \sqrt{z_{0}^{2}-4 m}}{2 m}$ are the roots of $m \xi^{2}-z_{0} \xi+1=0$ with $\left|r_{0}\right| \geq 1$, $\left|r_{i}\right| \leq 1$ and

$$
\xi=\frac{z \pm \sqrt{z^{2}-4 m}}{2 m} \text { with }|\xi| \leq 1
$$

The last term in equation (9) has singularities at $\xi=0$ and $\xi \doteq r_{1}$. inside the unit circle in the $\xi$ plane. For this case, let

$$
\phi^{*}=-\operatorname{lnm}-\ln \left(\xi-r_{0}\right)-\ln \left(1-r_{i} \bar{\xi}\right)
$$

so that

$$
\phi\left(z, \bar{z}, z_{0}\right)=\hat{\phi}+\phi^{*}=\ln \left(1-r_{i} / \xi\right)-\ln \left(1-r_{i} \bar{\xi}\right)
$$

This function vanishes on the unit circle $|\xi|=1$ (and therefore on the elliptical cutout in the $z$ plane) and satisfies the other properties required of the augmented kernel.

Single valued displacement on cutout boundary. The only nonzero displacement $u_{3}$ must be single valued on the cutout buundary, $1 . e$. it is required that

$$
\underset{\partial \mathrm{B}_{1}}{\mathrm{du}_{3}}=0
$$

This equation can be written in terms of the stress function $\Phi$ hy extending the analysis of Sokolnikoff [18] for the torsion of hollow beams, to this nonelastic case. The resulting equation is

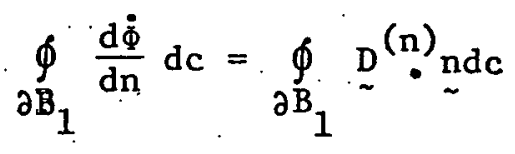


where $\mathfrak{n}$ is the unit outward normal to the cutout surface (Fig. 2) and

$$
D_{1}^{(n)}=2 G \dot{\varepsilon}_{32}^{n}, D_{2}^{(n)}=-2 G \dot{\varepsilon}_{31}^{\cdot n}
$$

Note. that $\underset{\nabla}{\nabla} \underline{\sim}^{(n)}=C^{(n)}$.

\section{Integral equations for stresses and tractions}

Integral Equations. The time-histories of the stress components $\sigma_{31}$ and $\sigma_{32}$ are of primary interest in this analysis. Thus, it is convenient to write integral equations directly for the stress rates using differentiated versions of the kernel $\phi$. For a body with an elliptic cutout (Fig. 3), the equations for the rates of stress are (with. j, $k=1,2$ )

$$
\begin{aligned}
2 \pi \dot{\sigma}_{3 j}(p) & =\oint_{\partial B_{2}} H_{3 j}(p, Q) c(Q) d c_{Q} \\
& +\int_{B} H_{3 j}(p, q) c^{(n)}(q) d A_{q} \\
& -\int_{\partial B_{1}} H_{3 j}(p, Q) D_{K}^{(n)}(Q) n_{K}(Q) d c_{Q}
\end{aligned}
$$

where the augmented kernels $\mathrm{H}_{3 j}$ are

$$
\begin{aligned}
& \mathrm{H}_{31}=\operatorname{Re}\left[\frac{\partial \phi}{\partial x_{2}}\left(z, \bar{z}, z_{0}\right)\right]=\operatorname{Im}\left(\frac{\partial \phi}{\partial z}-\frac{\partial \phi}{\partial z}\right) \\
& H_{32}=-\operatorname{Re}\left[\frac{\partial \phi}{\partial x_{1}}\left(z, \bar{z}, z_{0}\right)\right]=-\operatorname{Re}\left(\frac{\partial \phi}{\partial z}+\frac{\partial \phi}{\partial \bar{z}}\right)
\end{aligned}
$$

with $\phi$. given in equation (10).

The first two terms in equation (14) are analogous to equation (6) and the last term, which represents a layer of concentration $n \cdot D^{(n)}$ on the cutout boundary $\partial \mathrm{B}_{1}$, is necessary for obtaining single valued displacements on $\partial \mathrm{B}_{1}$. This is proved in the next subsection. 
The boundary conditions for the problem are specified in terms of traction rates on the outer boundary $\partial \mathrm{B}_{2}$. The traction rates are obtained from equation (14) by taking the limit as $\mathrm{P}$ in $\mathrm{B}$ approaches a point $\mathrm{P}$ on $\partial_{2} \cdot$ In taking this limit, care must be taken to obtain the residues from the singular kernels. It can be shown that for the rates of stress

if

$$
2 \pi \dot{\sigma}_{3 j}\left(p^{*}\right)=h\left(p^{*}\right)
$$

then $\quad 2 \pi \dot{\sigma}_{3 j}\left(P^{*}\right)=h\left(P^{*}\right)+\pi t_{j}\left(P^{*}\right) C\left(P^{*}\right)$

where $\mathrm{p}^{*}$ is infinitesimally close to $\mathrm{P}^{*}, \partial \mathrm{B}_{2}$ is locally smooth at $\mathrm{P}^{*}$ and $t_{j}\left(P^{*}\right)$ are the components of the unit (anticlockwise) tangent vector to $\partial \mathrm{B}_{2}$ at $P^{*}$. The function $h\left(P^{*}\right)$ is an abbreviation for the right hand side of equation (14). This implies that the residue is zero for the traction rate and equals $c(P) / 2$ for the stress rate component $\dot{\sigma}_{3 c}$ tangential to the boundary. The traction rate therefore, for a point $P$ on $\partial B_{2}$ where it is locally smooth, is; from equation (14) (with j, $k=1,2$ )

$$
\begin{aligned}
2 \pi \dot{\tau}(P) & =\oint_{\partial B_{2}} H_{3 j}(P, Q) n_{j}(P) C(Q) d c_{Q} \\
& +\int_{B} H_{3 j}(P, q) n_{j}(P) C C^{(n)}(q) d A_{q} \\
& -\oint_{\partial B_{1}} H_{3 j}(P, Q) n_{j}(P) D_{K}^{(n)}(Q) n_{K}(Q) d c_{Q}
\end{aligned}
$$

The boundary. integrals in the above must be interpreted in the sense of Cauchy principal values. It can be proved that $\mathrm{H}_{31}$ and $\mathrm{H}_{32}$, with an arbitrary source point, are independent of the position of the field point provided it lies on the elliptic cutout. This makes the last terms in equations (14) and (17) considerably easier to evaluate. 
Proof of single valued displacements on elliptic cutout boundary. The normal derivative of $\dot{\Phi}$ at a point $P$ an $\partial B_{1}$ (see Fig. 2) from equation (14), is

$$
\begin{aligned}
2 \pi \frac{\mathrm{d} \dot{\Phi}}{\mathrm{dn}}(\mathrm{P}) & =2 \pi\left(\dot{\sigma}_{31} \mathrm{n}_{2}-\dot{\sigma}_{32} \mathrm{n}_{1}\right) \\
& =\oint_{\partial \mathrm{B}_{2}} \operatorname{Re}\left[\frac{\mathrm{d} \phi}{\mathrm{dn}}(P, Q)\right] \mathrm{C}(\mathrm{Q}) \mathrm{dc} Q \\
& +\int_{\mathrm{B}} \operatorname{Re}\left[\frac{\mathrm{d} \phi}{\mathrm{dn}}(P, q)\right] \mathrm{C}^{(\mathrm{n})}(\mathrm{q}) \mathrm{dA} \mathrm{q}_{\mathrm{q}} \\
& +\oint_{\partial \mathrm{B}_{1}} \operatorname{Re}\left[\frac{\mathrm{d} \phi}{\mathrm{dn}}(P, Q)\right] \mathrm{D}_{\mathrm{K}}^{(\mathrm{n})}(Q) \mathrm{n}_{\mathrm{K}}(\mathrm{Q}) \mathrm{dc} \mathrm{C}_{Q}
\end{aligned}
$$

the positive sign on the last term being a consequence of the fact that $\mathbf{n}$ is the outward normal to $\partial \mathrm{B}_{1}$. The normal derivatives of the kernel $\phi$ are evaluated at the source point $P$ on $2 B_{1}$. There is no extra term due to a residue in this case.

The proof of equation (12) for single valued displacements on $\partial \mathrm{B}_{1}$ rests on the fact that, if the cutout is elliptic

$$
\oint_{\partial B_{1}} \operatorname{Re}\left[\frac{d \phi}{d n}(P, q)\right] d c_{P}=\left\{\begin{array}{cl}
0 & \text { if } q \text { is outside } \partial B_{1} \\
2 \pi & \text { if } Q \text { is on } \partial B_{1}
\end{array}\right.
$$

Proof of equation (19) is sketched briefly below. The above result is also true in the limiting cases when the cutout is a circle or a line crack. If a source point lies on the elliptical cutout $\partial B_{1}$

$$
\begin{aligned}
& z=(1+m) \cos \alpha+i(1-m) \sin \alpha \\
& \xi=e^{-i \alpha}
\end{aligned}
$$

where

$$
0 \leq \alpha \leq 2 \pi
$$


Let $n_{1}$ and $n_{2}$ be the components of the outward normal to $\partial B_{1}$ at some point on it. Then

$$
\tilde{n}=n_{1}+i n_{2}=\frac{e^{i \alpha}-m e^{-i \alpha}}{\sqrt{1+m^{2}-2 m \cos 2 \alpha}}
$$

and a line element on the ellipse

$$
d c=\sqrt{1+m^{2}-2 m \cos 2 \alpha} d \alpha
$$

Let an arbitrary field point $z_{0}$ yield the root (see below equation (9))

$$
r_{i}=B e^{-i \theta},\left(\begin{array}{llll}
B=1 & \text { if } z_{0} \text { is on } \partial_{1} \\
\beta<1 & \text { if } z_{0} \text { is outside } \partial B_{1}
\end{array}\right)
$$

Using all the above equations and equation (10) for $\phi\left(z, \bar{z}, z_{0}\right)$,

$$
\begin{gathered}
\operatorname{Re}\left[\frac{\mathrm{d} \phi}{\mathrm{dn}}(\mathrm{p}, \mathrm{q})\right]_{\because} \mathrm{dc}=\operatorname{Re}\left[\frac{\partial \phi}{\partial z} \tilde{\mathrm{n}}+\frac{\partial \phi}{\partial \bar{z}} \overline{\tilde{n}}\right] d c_{P} \\
=\frac{2(1-\delta \cos (\alpha-\theta))}{\delta^{2}-2 \delta \cos (\alpha-\theta)+1} \mathrm{~d} \alpha
\end{gathered}
$$

where $\delta=1 / \beta \geq 1, \ldots, \overline{\tilde{n}}=n_{1}-i n_{2}$ and $P$ is on $\partial B_{1}$

$$
\text { Now, with } \psi=\alpha-\theta
$$

$$
\int_{0}^{2 \pi} \frac{2(1-\delta \cos \psi)}{\delta^{2}-2 \delta \cos \psi+1} d \psi=\left\{\begin{array}{ccc}
0 & \text { if } \delta>1\left(z_{0} \text { outside } \partial \mathrm{B}_{1}\right) \\
2 \pi & \text { if } \delta=1\left(z_{0} \text { on } \partial \mathrm{B}_{1}\right)
\end{array}\right.
$$

which proves equation (19). 
Finally, integrating both sides of equation (18) around $\partial \mathrm{B}_{1}$, and evaluating first the integrals around $\cdot \partial \mathrm{B}_{1}$ in the resulting double integrals on the right hand side of equation (18),

$$
2 \pi \oint_{\partial B_{1}} \frac{\mathrm{d} \dot{\Phi}}{\mathrm{dn}} \mathrm{dc}=2 \pi \oint_{\partial \mathrm{B}_{1}} \mathrm{D}_{\mathrm{K}}^{(\mathrm{n})} \mathrm{n}_{\mathrm{K}} \mathrm{dc}
$$

which proves equation (12). 


\section{NUMERICAL IMPLEMENTATION}

\section{Discretization of equations}

The outer boundary of the body, $\partial_{2} \mathrm{~B}_{2}$ (Fig. 3), is divided into $\mathrm{N}_{2}$ straight boundary elements using $\mathrm{N}_{b}\left(\mathrm{~N}_{\mathrm{b}}=\mathrm{N}_{2}\right)$ boundary nodes and the inner boundary $\partial \mathrm{B}_{1}$ into $\mathrm{N}_{1}$ straight boundary segments. The interior of the body, $B$, is divided, into $n_{i}$ triangular internal elements. A discretized version of equation (17) is

$$
\begin{aligned}
& 2 \pi \dot{\tau}\left(P_{M}\right)=\sum_{N_{2}} \int_{\Delta c_{i}} H_{3 j}\left(P_{M}, Q\right) n_{j}\left(P_{M}\right) C(Q) d c_{Q} \\
& +\int_{i} \int_{\Delta A_{i}} H_{3 j}\left(P_{M}, q\right) n_{j}\left(P_{M}\right) C^{(n)}(q) d A_{q} \\
& -H_{3 j}\left(P_{M}, \hat{Q}\right) n_{j}\left(P_{M}\right) \sum_{N_{1}} \int_{\Delta c_{i}} D_{K}^{(n)}(Q) n_{K}(Q) d c_{Q}
\end{aligned}
$$

where $\tau\left(P_{M}\right)$ are the traction components at the point $P$ which coincides with the node $M$ at the center of a segment on $\partial B_{2}, \Delta c_{i}$ and $\Delta A_{i}$ are boundary and internal elements respectively and $\hat{Q}$ is any point on $\partial B_{1}$.

A very simple numerical scheme is used in which the concentrations $C$ are assumed to be piewise uniform on each boundary segment with their values assigned at the nodes which lie at the centers of each segment. The nonelastic strain rates $\dot{\varepsilon}_{3 j}^{n}$ are interpolated linearly over each triangular internal element so that $c^{(n)}$ in uniform within each element. For the problems considered in this section, the last integral in equation (17) can be shown to vanish, i.e., in these cases

$$
\oint_{\partial \mathrm{B}_{1}} \underline{\mathrm{D}}^{(\mathrm{n})} \text { ndc }=0
$$

by virtue of the stress pattern and the constitutive model. Hence the last term in equation (20) is omitted in these calculations. In any case, in general, this term requires the evaluation of a known integral on ${ }^{\partial B_{1}}$ at each time step. 
The integrals of $\mathrm{H}_{3 \mathrm{j}}$ on boundary elements are evaluated analytically for the singular and by Gaussian quadrature for the regular portions. Integrals of $\mathrm{H}_{3 \mathrm{j}}$ on triangular internal elements are obtained by Gaussian quadrature. This is adequate in these problems with the source points lying on the vertices of the triangles.

Substitution of the piecewise uniform source strengths into equation (20) and carrying out of the necessary integrations leads to an algebraic system of the type

$$
\{\dot{\tau}\}=[A]\{C\}+\{d\}
$$

The coefficients of the matrix [A] contain boundary integrals of the kerne1s. The traction rates are prescribed, the vector $\{d\}$ contains the contributions from the area integral and the vector $\{C\}$ the unknown source strengths at the boundary nodes. The dimension of the unknown vector $C$ depends only on the number of boundary elements on $\because \partial \mathrm{B}_{2}$ :

Equation (14) for the stress rates at an internal point $p$ are discretized in a similar fashion.

\section{Solution strategy}

The power law creep constitutive model, used to describe material behavior, has the form [19]

$$
\begin{aligned}
& \dot{\varepsilon}_{i j}=\dot{\varepsilon}_{i j}^{c}+\ddot{\varepsilon}_{i j}^{n} \\
& \dot{\varepsilon}_{i j}^{n}=\frac{3}{2} \frac{\dot{\varepsilon}^{n}}{\sigma} \varepsilon_{i j} \\
& \dot{\varepsilon}^{n}=\dot{\varepsilon}_{c}\left(\frac{\dot{\sigma}}{\sigma_{c}}\right)^{m}
\end{aligned}
$$


where the stress and strain rate invariants are defined as

$$
\sigma=\sqrt{(3 / 2) s_{i j} s_{i j}}, \quad \dot{\varepsilon}^{n}=\sqrt{(2 / 3) \dot{\varepsilon}_{i j}^{n} \dot{\varepsilon}_{i j}^{n}}
$$

with $s_{i j}$ the deviatoric part of the stress tensor $\sigma_{i j}, \dot{\varepsilon}_{c}$ and $\sigma_{c}$ the reference strain rate and reference stress rate, respectively, and $m_{0}$ the index of the power law.

The solution strategy is as follows. The initial stress field is obtained by solving the corresponding elastic problem. The initial rates of the nonelastic strains are obtained from the constitutive model (22). The vector $\frac{\mathrm{d}}{\mathrm{d}}$ in equation (21) is calculated next, and this, together with the prescribed rates of boundary tractions, are used to calculate the initial distribution of concentration,$C$ on $2 B_{1}$. These concentrations are now used in the discretized version of equation (14) to calculate the initial stress rates throughout the body. These rates are used to determine the values of the variables after a small time interval $\Delta t$, and so on, and in this way the time histories of the relevant variables are obtained. Time integration is carried out by an Euler type step-wise procedure with automatic time-step control. This method is described in refs. [15, 20]. 
NUMERICAL RESULTS AND DISCUSSION

\section{Material parameters}

The material parameters for stainless steel at $400^{\circ} \mathrm{C}$, used in these numerical calculations, are [19]

$$
\begin{aligned}
& \mathrm{G}=9.4 \times 10^{6} \mathrm{psi} \\
& \dot{\varepsilon}_{\mathrm{c}}=0.277 \times 10^{-3} \mathrm{~s}^{-1} \quad \sigma_{\mathrm{c}}=0.1806 \times 10^{6} \mathrm{psi} \\
& \mathrm{m}_{\mathrm{o}}=7
\end{aligned}
$$

\section{Numerical results}

Elastic solution. In order to check the program, an elastic solution is first obtained for a finite plate with a line crack $(m=1)$ loaded in anti-plane shear. The stress distribution has a pattern which makes it possible to deduce the stresses in the entire plate from the stresses in the first quadrant.: Thus only a quarter of the plate (the first quadrant: in this case) is discretized. Eight boundary nodes are placed uniformly along the edges of the plate in the first quadrant. The elastic solution for $\sigma_{32}$, obtained numerically, has the proper square root singularly near the crack tip. The calculated value of the stress intensity factor is within 5 per cent of the analytical solution for an infinite plate with a crack [21].

Nonelastic solutions. A stationary crack is modelled as an ellipse with axis ratio 19.9. $(m=0.99)$ except for the results shown in Fig. 8 where $a / b=1999(m=$ 0.999.). The cracked plate is loaded in anti-plane shear as shown in Fig. 4a. Once again, only a quarter of the plate is discretized. Twenty boundary nodes are distributed uniformly along the edges of the plate in the first quadrant. 
High concentrations of stresses, and consequently strain rates, are present near the crack tips. Therefore, only the region very near the tip of the major axis of the ellipse is discretized into internal elements (Figs. $4 \mathrm{a}$ and $4 \mathrm{~b}$ ) and it is assumed that all the nonelastic contribution to the stress rates in equation (14) is obtained from this discretized area. (As stated earlier, the last term in equation (14) is zero in these problems). Thus, in addition to the fact that the number of unknowns depend only on the number of boundary elements, evaluation of area integrals by quadrature is greatly simplified by the fact that only a small portion of this area needs to be discretized. Input time as well as computational time is reduced since relatively few internal elements are necessary. These simplifications are important advantages of the boundary element method relative to the finite element method and lead to substantial savings in cost.

The decrease of stress concentration at the crack tip as a function of time, for the case of constant remote stress, is shown in Fig. 5. The decrease is due to flow of the material near the crack tip and consequent accommodation of stress. The redistribution of stress concentration with time along the line $x_{2}=0$ near the crack tip is shown in Fig. 6. The near field stress distribution tends to even out with time.

Figure 7 shows the drop in stress concentration at the crack tip as functions of time for two different rates of remote loading. Stress relaxation is less at the higher loading rate since there is less time for nonelastic flow in this case. Also, very little stress relaxation takes place at very short times while the remote stress is building up. The stress concentration at large values of the remote stress is of the same order as the long time values from Fig. 5 for the constant remote stress case.

Finally, Fig. 8 shows the drop in stress concentration at the crack tip when the crack is modelled as a very sharp ellipse of axis ratio 1999. There is no numerical difficulty in carrying out this calculation since, as stated before, 
the effect of the crack is included in the kernel and the only unknown quantities in the algebraic equations are on the outer boundary of the plate. In this case, a different internal mesh is used. This mesh is given in the Appendix.

The numerical results presented in this section are stable. A typical c.p.u. time on an IBM $370 / 168$ computer is 179 seconds for the results shown in Fig. 8 for $\mathrm{m}=.999$. 


\section{CONCLUSIONS}

The boundary element formulation with augmented kernels used in this paper is very efficient for the analysis of stresses in cracked plates undergoing inelastic deformation. The primary reasons for this claim are as follows.

1) The proper boundary conditions on the crack boundary are satisfied in an implicit manner.

2). The only unknowns appear on the outside boundary of the plate.

3) Internal discretization is required over only a small region near the crack tip. Area integrals over this region involve known integrands at every time step and these are evaluated by quadrature over relatively few internal elements. 42. No apriori assumptions regarding the stress distribution near the crack tip need to be made.

The method yields stable numerical results for different histories of remote loading even for a crack modelled as a very thin ellipse of aspect ratio near 2000 . 
ACKNOWLEDGEMENTS

This research was supported by contract No. EG-77-S-02-2733 of the U.S. Department of Energy with Cornell University. 


\section{REFERENCES}

1. R. Shaw, et al. (ed.), Innovative Numerical Analysis for the Applied Engineering Sciences, University Press of Virginia, Charlottesville, Virginia (1980).

2. P.K. Banerjee and R. Butterfield (ed.), Developments in Boundary Element Methods I, Applied Science Publishers, London (1979).

3. C.A. Brebbia (ed.), Recent Advances in Boundary Element Methods, Pentech Press, London, Plymouth (1978).

4. T.A. Cruse and F.J. Rizzo (ed.), Boundary Integral Equation Method: Computational Applications in Applied Mechanics, American Society of Mechanical Engineers, New York (1975):

5. V. Kumar and S. Mukherjee, A boundary-integral equation formulation for timedependent inelastic deformation in metals. Int. J. Mech. Sci. 19, 713 (1977).

6. S. Mukherjee and V. Kumar, Numerical Analysis of time-dependent inelastic deformation in metallic media using the boundary integral equation method.

ASME J. Appl. Mech. 45, 785 (1978).

7. M. Morjaria and S. Mukherjee, Improved boundary-integral equation method for time-dependent inelastic deformation in metals. Int. J. Num. Meth. Engng. $15,9.7$ (1980).

8. M. Morjaria, V. Sarihan and S. Mukherjee, Comparison of boundary element and finite element methods in two-dimensional inelastic analysis. Res. Mechanica, 1,3 (1980).

9. M. Morjaria and S. Mukherjee, Inelastic analysis of transverse deflection of plates by the boundary element method. ASME J. Appl. Mech. 47, 291 (1980).

10. S. Mukherjee, Applications of the boundary element method in time-dependent inelastic deformation, in Innovative Numerical Analysis for the Applied Engineering Sciences, (R. Shaw, et al. (ed.)); University Press of Virginia, Charlottesville, Virginia (1980).

11. T.A. Cruse, Two dimensional bie fracture mechanics analysis, in Recent Advances in Boundary Element Methods (C.A. Brebbia ed.) Prentech Press, London, P1ymouth; 167 (1978).

12. A. Mirtohamad-Sadegh and N.J. Altiero, A boundary-integral approach to the problem of an elastic region weakened by an arbitrarily shaped hole. Mech. Res: Corm. 6, 167 (1979).

13. A. Mir-Mohamad-Sadegh and N.J. Altiero, Solution of the problem of a crack in a finite plane region using an indirect boundary-integral method. Engng. Frac. Mech. 11, 831 (1979).

14. S, Mukherjee and M. Morjaria, A boundary element formulation for planar timedependent inelastic deformation of plates with cutouts. Int. J. Solids Structures.' (In Press). 
15. M. Morjaria and S. Mukherjee, Numerical analysis of planar, time-dependent inelastic deformation of plates with cracks by the boundary element method. Int. J. Solids Structures, (In Press).

16. L.M. Milne-Thomson, Theoretical Aerodynamics (4thed.), Macmillan, New York (1966).

17. H. Riedel, Cracks loaded in anti-plane shear under creep conditions, $Z$. Metallkde, 69, 775 (1978).

18. I.S. Sokolnikoff, Mathematical Theory of Elasticity, McGraw Hill (1956).

19. F.K. G. Odquist, Mathematical Theory of Creep and Creep Rupture, Oxford Clarendon Press (1966).

20. V. Kumar, M. Morjaria and S. Mukherjee, Numerical integration of some stiff constitutive models of inelastic deformation. ASME J. Engng. Mat. Tech. 102,92 (1980).

21. J.P. Benthem and W.T. Koiter, Asymptotic approximations to crack problems, in Mechanics of Fracture 1, G.C. Sih ed., Noordhoff, Leyden, 131 (1973).

22. S. Mukherjee and M. Morjaria, Boundary element analys is of time-dependent inelastic deformation of cracked plates loaded in anti-plane shear. DOE report: No, C00-2733-28:, Department of Theoretical and Applied Mechanics, Cornell University, Ithaca, NY (1980). 


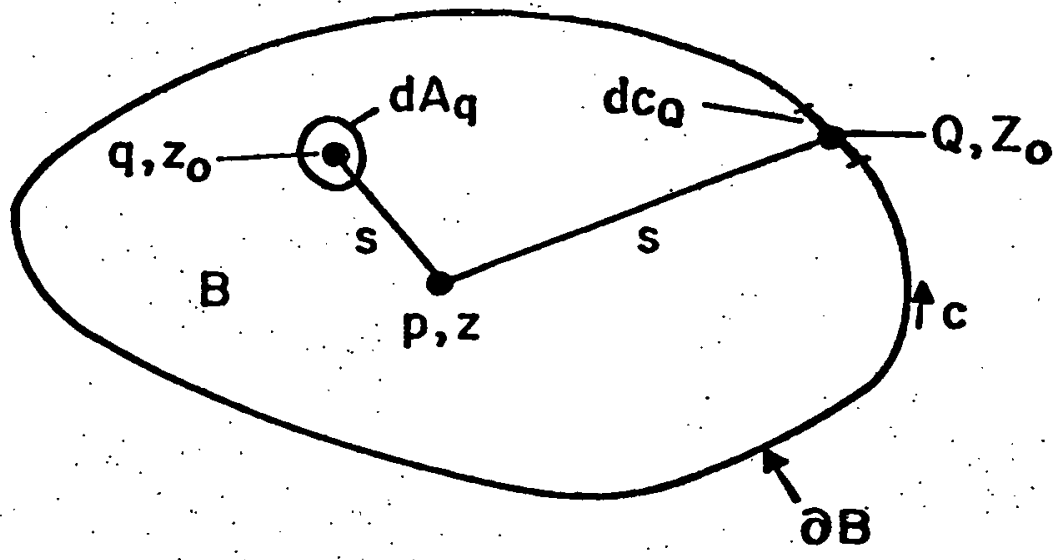

Figure 1. 


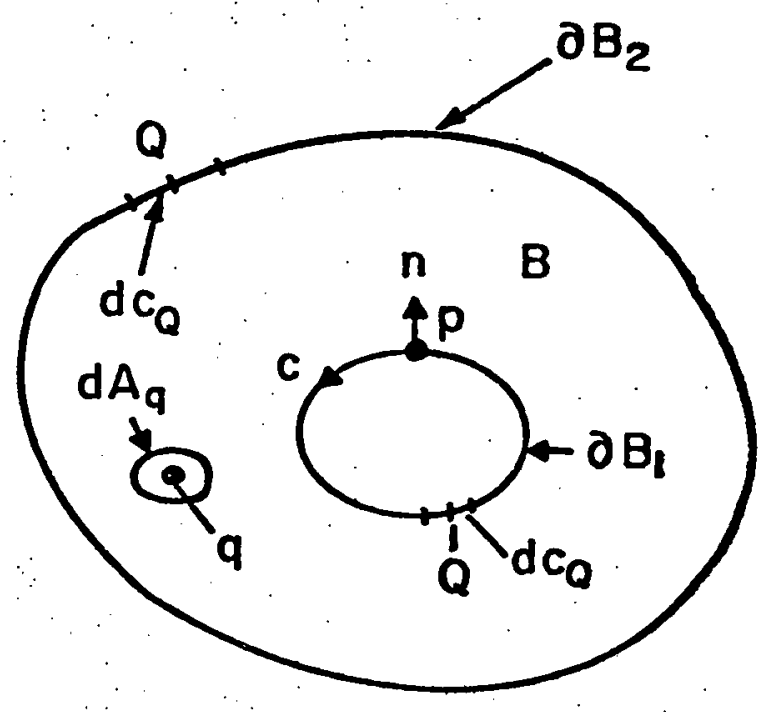

Figure 2. 


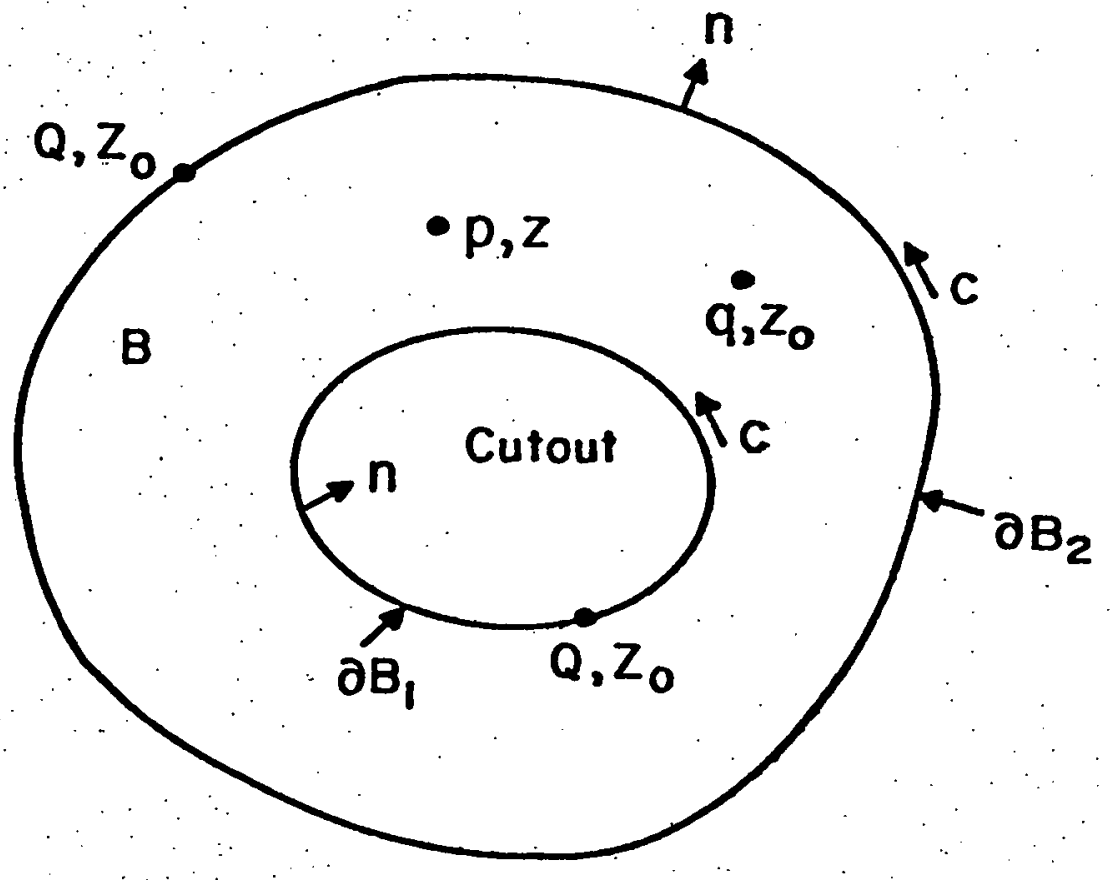

Figure 3. 


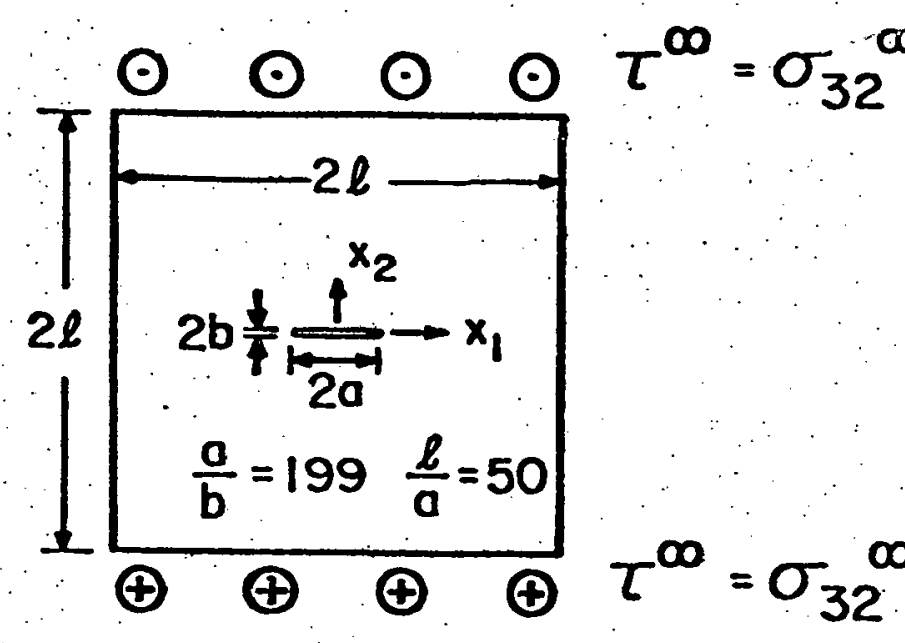

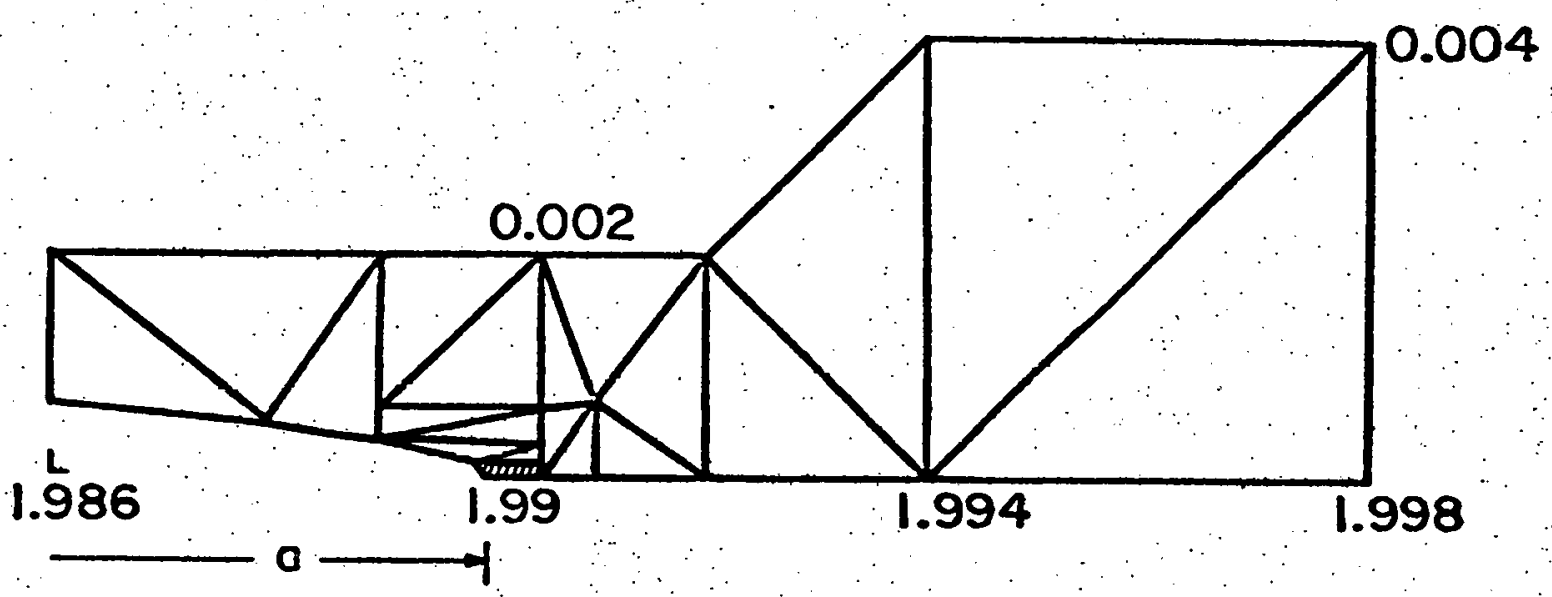

Figure 4(a). Internal elements for cracked plate loaded in antiplane shear. 


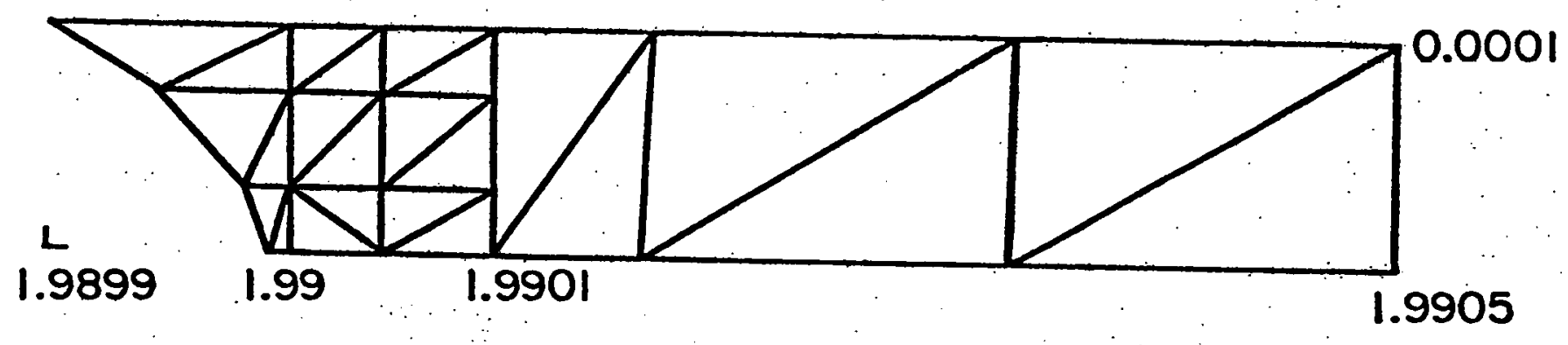

Figure $4(\mathrm{~b})$. Further details of figure $4(\mathrm{a})$. 


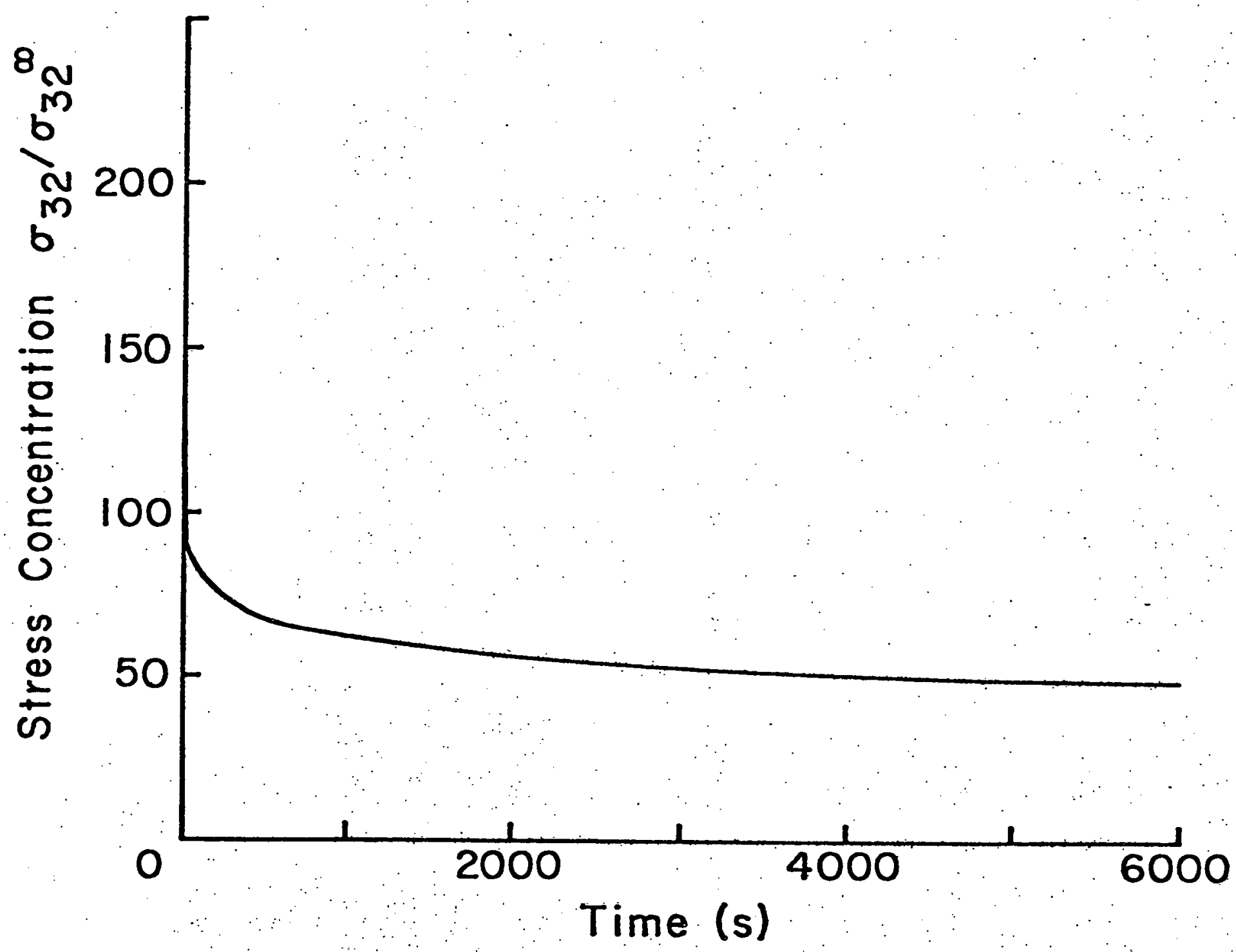

Figure.5. Stress concentration at crack tip as a function of time for constant remote stress $\sigma_{32}^{\infty}=1000 \mathrm{ps} 1, a / b=199$. Initial stress concentration $=204$. 


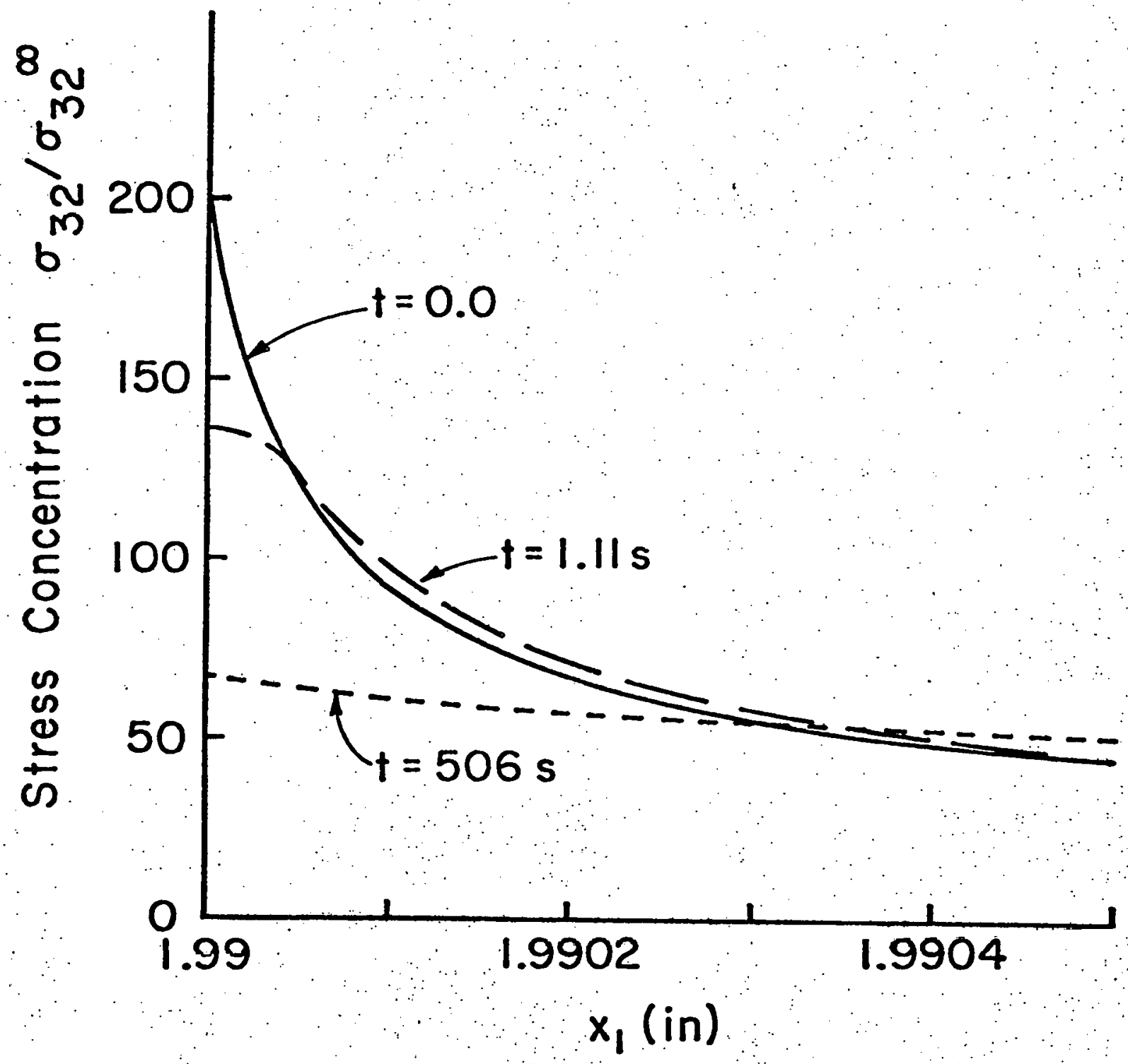

Figure 6. Stress redistribution along the line $x_{2}=0$ in cracked plate for constant remote stress $\sigma_{32}^{\infty}=1000 \mathrm{psi}, \mathrm{a} / \mathrm{b}=199$ 


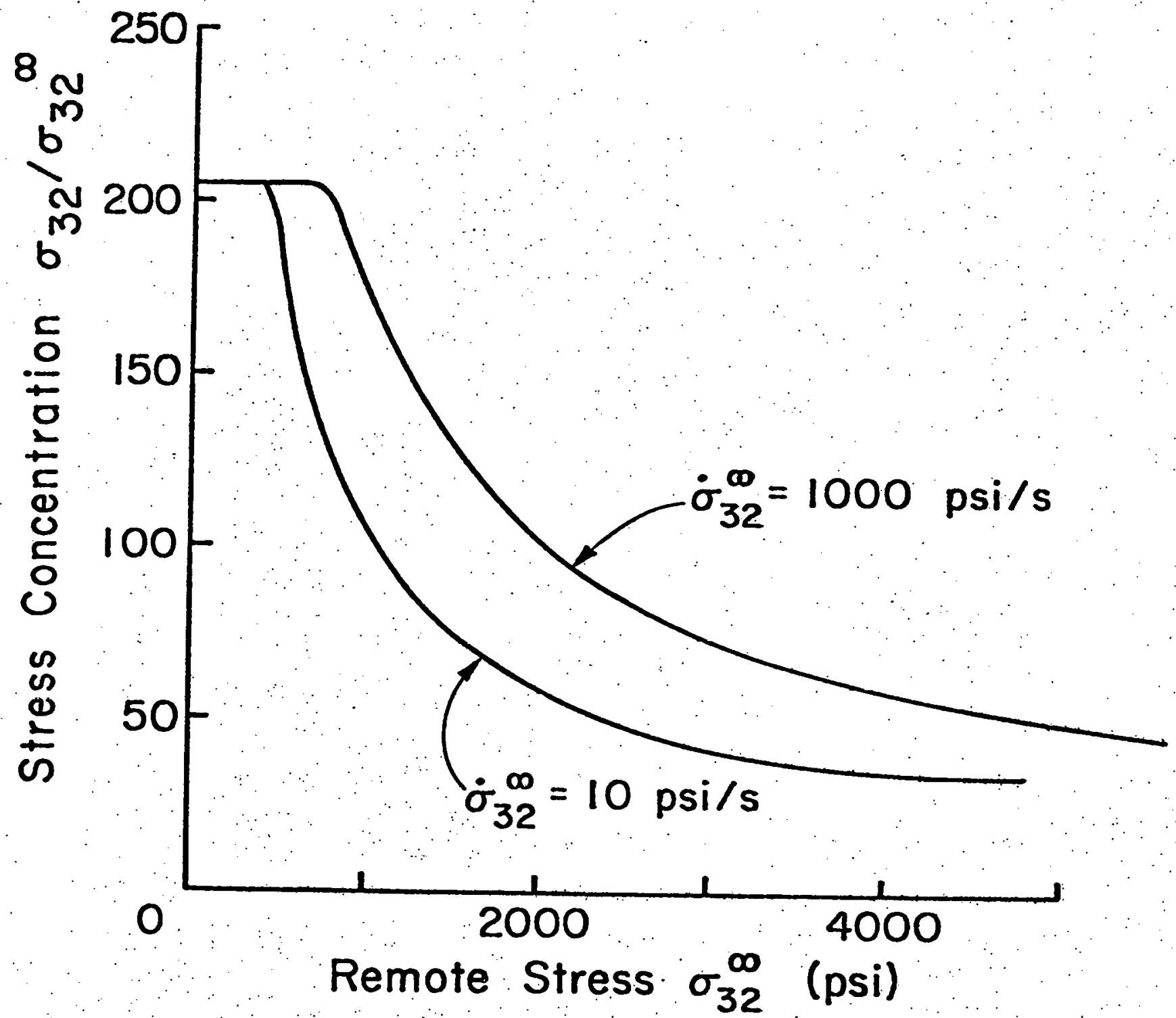

Figure 7. Stress concentration at crack tip as functions of remote stress for remote stress increasing at a constant rate $a / b=199$ 


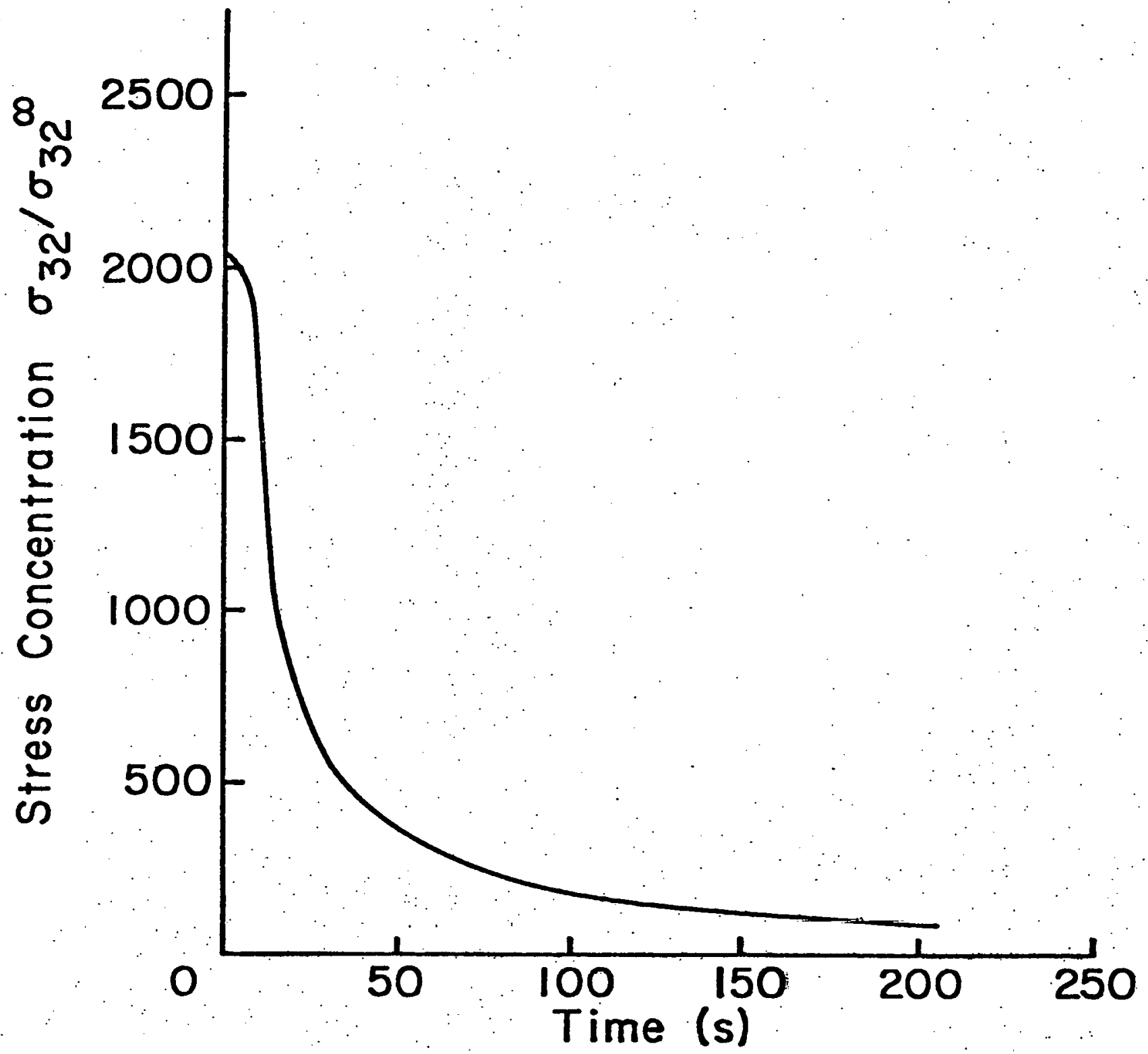

Figure 8. Stress concentration at crack tip as a function of time for coristant remote stress $\quad \sigma_{32}^{\infty}=1000$ psi, $a / b=1999$. 


\section{APPENDIX}

Internal element mesh for $a / b=1999$ used for results shown in Fig. 8 . 
(a)

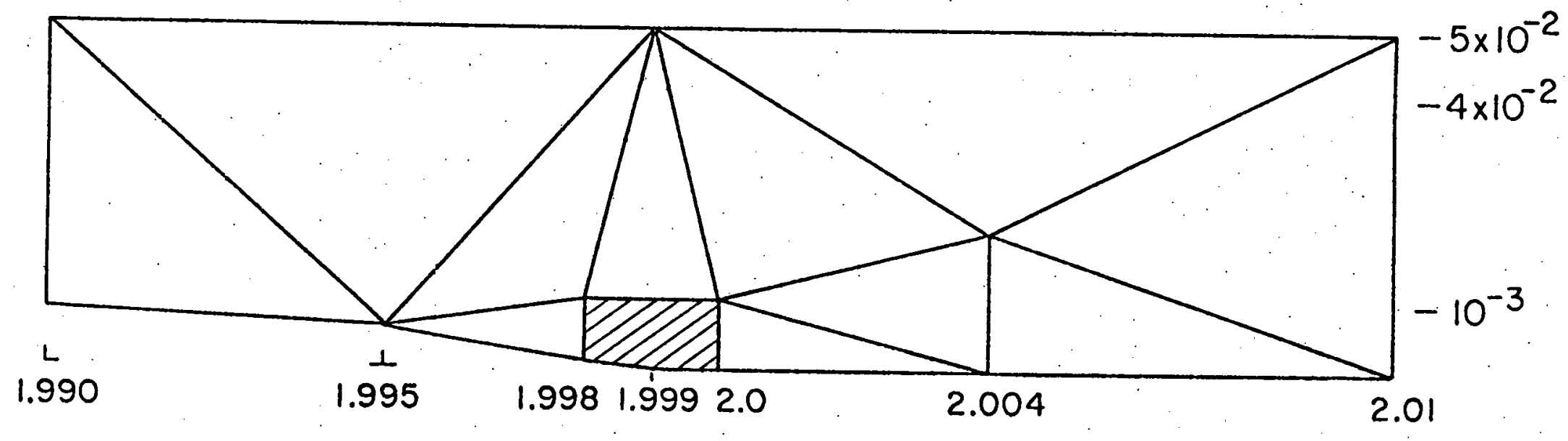


(b)

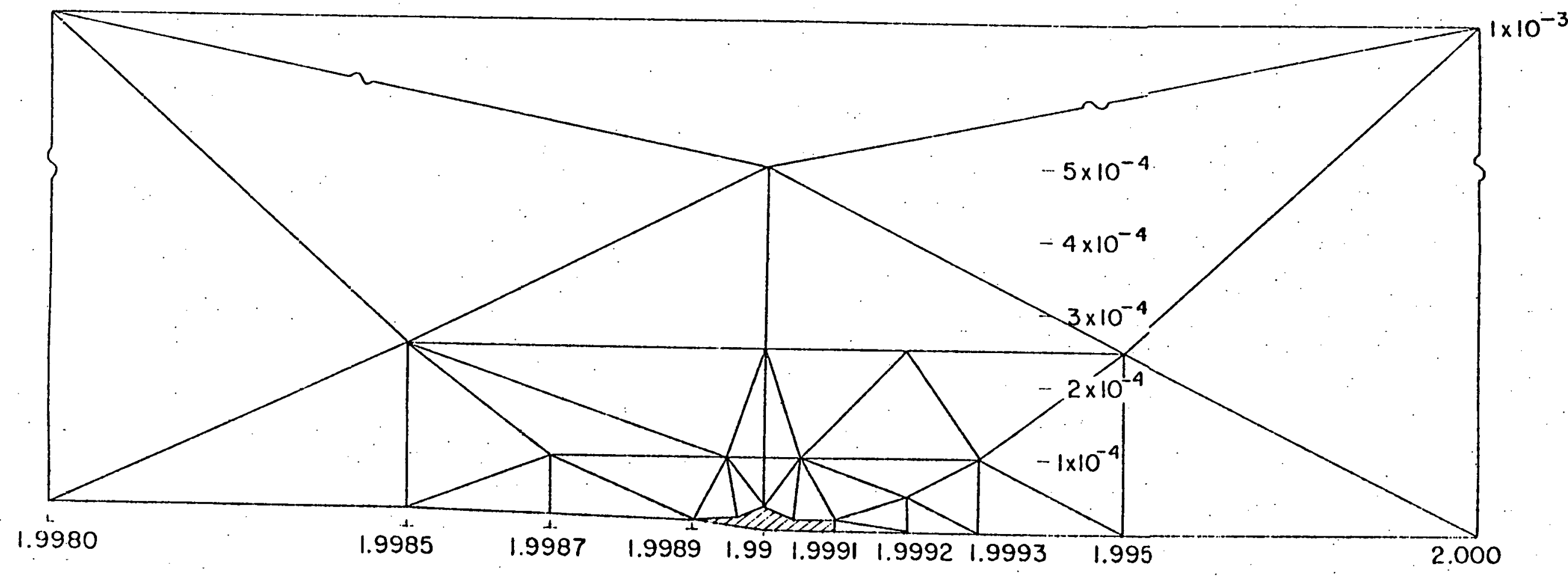


(c)

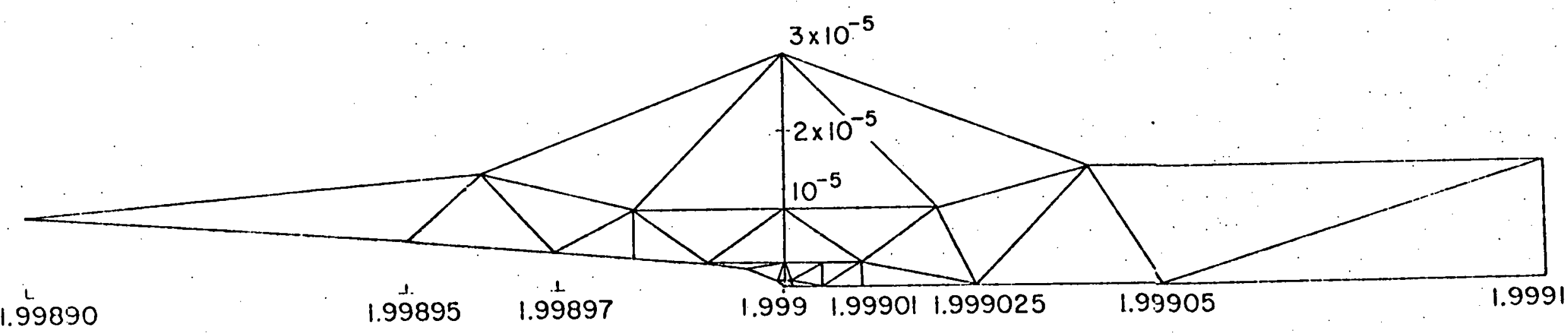


(d)

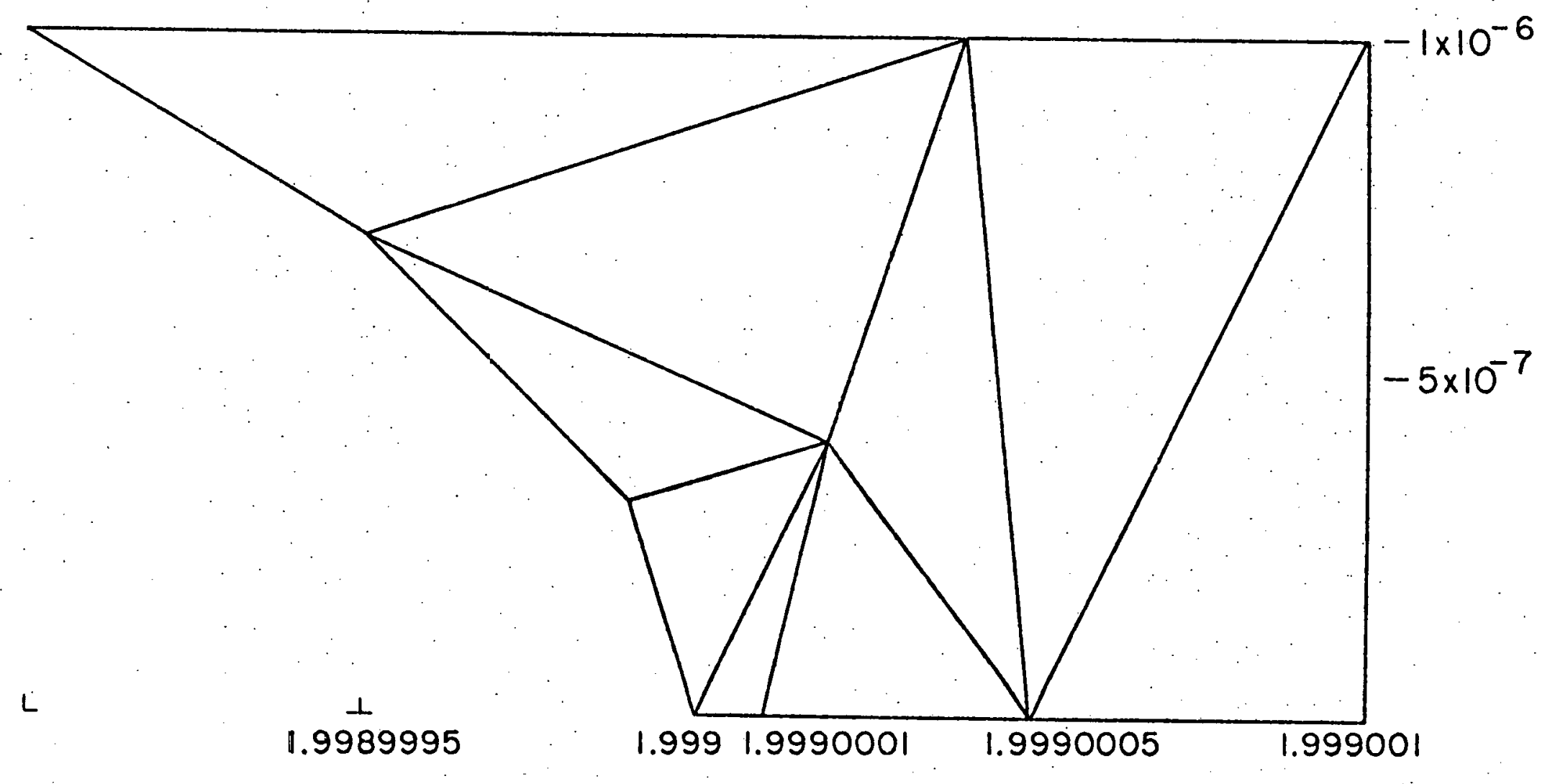

